Ann. Sci. forest., 1980, 37 (1), 37-51.

\title{
Essai d'interprétation écologique de la nématofaune de la forêt landaise ( ${ }^{1}$ )
}

\author{
C. SCOTTO LA MASSESE * ef A. BOULBRIA ** \\ avec la collaboration technique de MM. J. P. FRANKOWSKI, J. C. MINOT et R. VOISIN * \\ * Station de Recherches sur les Nématodes I.N.R.A., \\ 123, Bd F. Meilland 06602 Antibes \\ ** Laboratoire de Sylviculture ef d'Ecologie de la Pinède Landaise, I.N.R.A., \\ Pierrofon-Cestas, 33610 Gazinet
}

\begin{abstract}
Résumé
Une prospection réalisée de 1973 à 1976, dans le massif forestier des Landes, a révélé une faune nématologique d'une exceptionnelle originalité ef d'une certaine homogénéité.

L'originalité se traduit en particulier par l'existence quasi exclusive pour la france de deux genres appartenant aux sous-familles des Hoplolaiminae et des Criconematinae et par la présence de cinq espèces nouvelles de Tylenchides, Rotylenchus, Helicotylenchus, Hemicriconemoides, Criconema aquitanense Fies, 1965 et d'une espèce de Trichodorus.

L'homogénéité se traduit par une grande similarité de composition et de structure des nématocénoses de la plupart des sites échantillonnés qui renferment tous une ou plusieurs espèces caractéristiques des Landes. Or, il n'est pas possible d'expliquer par les interventions humaines cette dispersion tout à fait exceptionnelle sur une étendue de plus de dix mille $\mathrm{km}^{2}$.

Ces deux particularités semblent bien résulter des facteurs climatiques, des caractéristiques pédologiques et phytosociologiques très homogènes du massif landais.

C'est sans doute les particularités édaphiques et agropédologiques qui paraissent jouer un rôle prépondérant sur la nématofaune très originale de cette région.
\end{abstract}

\section{I. - Introduction}

La composition de la nématofaune est influencée par de nombreux facteurs d'ordre édaphique, climatique, phytosociologique, qui excercent un tri parmi les espèces endémiques et introduites.

Du fait de son homogénéité agropédologique et de son peuplement à base de pin maritime sur l'ensemble de son étendue, le massif landais, qui couvre dans le Sud-Ouest de la France un million d'hectares environ, constitue un bon modèle pour vérifier si l'unité synécologique de cette région est perceptible par l'analyse nématologique.

Cet inventaire, qui débute à peine, a pu être entrepris grâce au concours de nos collègues forestiers et à la compréhension de la D. G. R. S. T. qui nous a accordé un

(1) Etude préliminaire réalisée dans le cadre du contrat « Equilibre biologique et production en forêt landaise » financé par la Délégation Générale à la Recherche Scientifique et Technique (D.G.R.S.T.). 
soutien financier au sein du groupe de travail « Equilibre biologique et production en Forêt Landaise ».

Nous ne présentons ici que quelques considérations sur la nature ef la répartition des principaux groupes trophiques, en nous étendant quelque peu sur les espèces phytophages en raison même de l'hypothèse de travail à l'origine de la participation de la nématologie à l'étude des dépérissements du pin dans la région landaise.

Mais, notre ambition à plus longue échéance est d'analyser la composition spécifique de tous les groupes de nématodes ef spécialement ceux qui, moins inféodés au peuplement végétal, sont susceptibles d'être plus dépendants des conditions écologiques ef des caractéristiques édaphiques des milieux.

\section{II. - Description de la zone prospectée (*)}

Cette région de forme triangulaire est limitée par l'Océan à l'Ouest, par la Garonne et la Gironde à l'Est, la vallée de l'Adour au Sud. Elle s'étend sur la majeure partie du département des Landes, la moitié de la Gironde et le cinquième du Lot-etGaronne.

- Le climat est océanique, c'est-à-dire doux, humide, à pluviométrie assez uniformément répartie dans l'année.

- Les sols se sont développés sur des formations sableuses, d'origine fluviatile, reprises par le vent au cours de la période sèche qui a suivi la dernière glaciation wurmsienne. L'influence éolienne a pu agir jusqu'au Moyen-âge. Ces formations sableuses, d'épaisseur variable, reposent sur un substratum calcaire ou molassique vers l'Est et graveleux, sableux ou argileux à l'Ouest (ces sols, formés en période froide, appartiennent au groupe podzolique et se caractérisent donc par des $\mathrm{pH}$ très acides, une matière organique peu évoluée à $C / N$ élevé ; localement, une réévolution sous climat atlantique les a transformés en sols lessivés).

Le relief général, hors des dunes à la topographie très accusée, est du type monotone à pente faible vers l'Ouest, entaillé plus ou moins profondément par un réseau hydrographique peu hiérarchisé.

De ce bref aperçu on peut déduire les principaux types de « paysages » rencontrés

En bordure atlantique, la dune est la zone la plus mouvementée. Elle est constituée de sable presque pur à niveau d'eau en général très profond, mais pouvant affleurer dans les creux. Sa colonisation commence par un chiendent (Agropyrum junceum) au bord de la mer pour aboutir à la chênaie (Quercus spp.) dans les dunes les plus anciennes.

La lande, sans relief marqué, dont la végétation varie surtout avec la profondeur de la nappe phréatique qui parfois affleure à la surface du sol.

- Aspect phytosociologique (Fig. 1).

Grâce à sa plasticité ef aux soins dont il est l'objet, le pin maritime a pu être installé aussi bien dans le sable presque pur que dans les sols tourbeux. Son implantation commencée à la fin du XVIIIe siècle courrait 900000 hectares en 1900.

(*) La plupart des éléments de ce chapitre sont extraits de l'opuscule « La forêt landaise » de J. Guinaudeau, I.N.R.A., 1973 eł ont été revus par M. Gelpe que nous remercions bien vivement. 


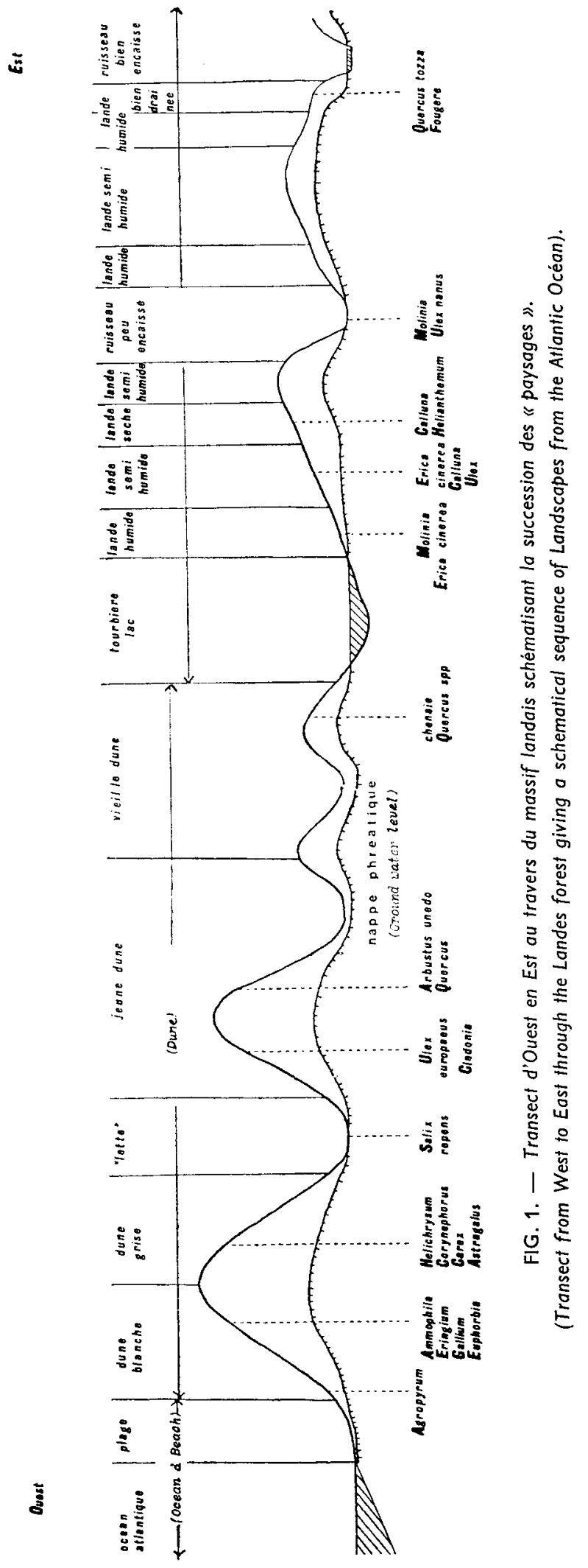


Sa régénération s'effectue très généralement par semis naturel dans la dune, mais il faut systématiquement faire appel au semis artificiel dans la lande (sauf après incendie).

Le sous-bois est influencé par la nature du sol et surtout par son hydromorphie, il peuł l'être également par un passé ou des pratiques culturales. Si l'on excepte les espèces xérophiles et halophiles qui s'installent en bordure des plages, il n'existe, comme le fait remarquer Demounem (1968), que peu d'espèces indicatrices de milieu. C'est donc surtout par leur abondance respective que les différents représentants de couvert végétal naturel donnent aux paysages leur apparence.

La succession des associations végétales, en allant des sites plus secs vers les plus humides, a été représentée dans la figure 1.

En ce qui concerne la lande, on trouve principalement en partant des sites les plus secs les espèces végétales suivantes classées par ordre de dominance décroissante :

- En lande sèche :

- Calluna vulgaris ef Helianthemum alyssoides (dominants).

Erica cinerea.

Quercus tozza.

- Ulex europaeus (dominant).

Calluna vulgaris.

Erica cinerea ef E. scoparia.

- Pteridium aquilinum (dominant).

Ulex europaeus.

Erica cinerea ef E. scoparia.

- En lande humide :

- Ulex nanus ef Erica scoparia (dominants).

Molinia caerulea.

Erica cinerea.

Erica tetralix.

Erica ciliaris.

- En lande très humide à plan d'eau superficiel :

- Molinia caerulea (dominant).

Alisma ranunculoides.

Schoenus nigricans (rare).

Ainsi, le pin maritime occupant à peu près tous les paysages, depuis la « jeune dune " et les seules différences dans le couvert végétal étant de nature quantitative plus que qualitative, les variations dans la composition de la nématofaune phytophage paraissent devoir être limitées aux conditions extrêmes de la séquence énumérée ci-dessus.

\section{III. - Méthodes de prélèvement}

L'étude ayant eu pour but de vérifier l'influence éventuelle de la nématofaune phytophage sur le développement du pin, la plupart des échantillons ont été réalisés 
par couple dans un certain nombre de placettes. Dans chaque couple un des sites est situé dans une zone où le développement est satisfaisant, l'autre dans un point où le pin est moins vigoureux, voire mort. Dans tous les cas les deux niveaux, 0 à $20 \mathrm{~cm}$ et 20 à $40 \mathrm{~cm}$ de profondeur, ont été analysés séparément.

Un certain nombre d'échantillons ont également été prélevés pour connaître la distribution de la nématofaune sur toute la surface du massif étudié et dans les forêts périphériques de pins maritimes (Carte I).

\section{CARTE 1}

Localisation et caractéristiques mésologiques des sites échantillonnés

(Location and characteristics of the sampling sites).

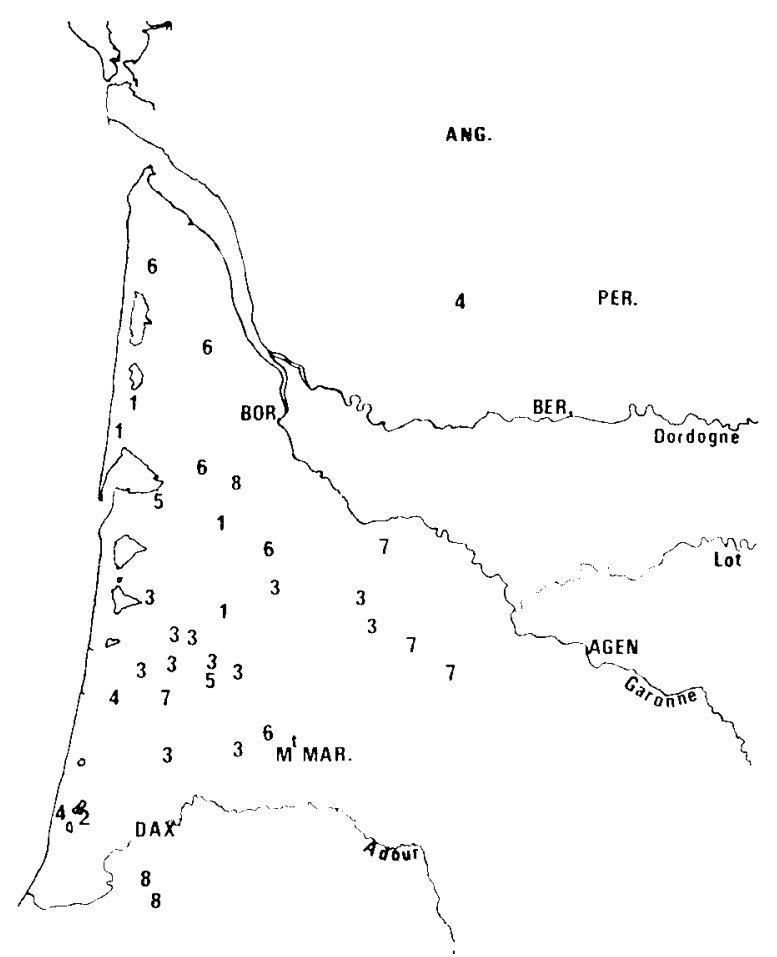

Newly established dune

Old dune

Dry heath

Well drained heath

Semi wet heath

Wef heath

Other samples

Other hosts

nières.

Une série d'analyses a en outre permis de préciser les fluctuations saison-

Enfin la distribution verticale est à l'origine de plusieurs séries d'échantillons. 


\section{IV. - Méthode d'estimation de la nématofaune}

Aucune espèce endoradiculaire n'ayant été trouvée dans le sol au cours de la prospection et de l'analyse préliminaire de racines de pin et des planies du sous-bois, tous les résultats ci-dessous mentionnés sont obtenus d'extractions du sol.

Celles-ci ont été faites par la méthode d'élutriation-centrifugation appliquée à des poids d'environ $1 \mathrm{~kg}$ de terre fine. Les chiffres ont été ramenés ensuite à des estimations pour $100 \mathrm{~g}$ de sol dans l'expression des résultats.

\section{V. - Résultats (tableau 1)}

Les nématodes identifiés ont été classés, selon leurs exigences trophiques, en quatre groupes :

- Les phytophages, analysés avec plus de détail, ci-dessous, en raison même de l'objectif de départ.

- Les mycophages, regroupant les Aphelenchus, les Aphelenchoides et une partie des Tylenchus.

- Les saprophages-bactériophages, représentés surtout dans cette région par les Cephalobidae, les Plectidae, les Chromadoridae, les Panagrolaimidae.

- Les créophages ou prédateurs comportent cinq genres : lotonchus, Miconchus, Mononchus, Tripyla ef Choanolaimus.

Certains nématodes, comme Ecphyadophora. Tylolaimophorus et plusieurs Dorylaimides, dont le régime alimentaire demeure inconnu, ont été également rencontrés et dénombrés.

\section{La nématofaune phytophage}

Les nématodes phytophages confèrent au massif landais une unité et une singularité accusées par rapport à la nématofaune déjà connue dans le reste de la France et de l'Europe.

Parmi les Tylenchina :

- Les Tylenchus et genres apparentés sont en faible nombre.

- Aucun Tylenchorhynchus n'a été trouvé.

- Les Heteroderidae y sont également absents.

- Les Hoplolaimidae constituent le groupe le plus important de la nématofaune. Toutefois les analyses n'ont pas révélé de Pratylenchus presque toujours présents par une ou plusieurs espèces dans les sols cultivés en France. Ce sont les Hoplolaiminae qui donnent à la nématofaune phytophage beaucoup de son originalité. Quatre espèces ont été décelées. Une nouvelle espèce du genre Scutellonema non encore signalée en plein air en Europe (mais trouvée sur carottes dans le Centre Est de la France, il y a une dizaine d'années). Cette espèce, dont la description est en cours est caractérisée par neuf à dix anneaux labiaux. Elle existe dans la quasi totalité des 
sites échantillonnés, sauf toutefois dans les dunes de formation récente où le sous-bois est pratiquement inexisiant.

Le nombre d'individus peuł dépasser $1000 / 100 \mathrm{~g}$ de sol. Il est presque toujours plus abondant dans les horizons de surface el absent au-dessous de $40 \mathrm{~cm}$.

Un essai, destiné à vérifier les rôles respectifs du sous-bois et du pin maritime sur la composition de la faune nématologique, confirme que le pin ne permet pas à cette espèce de se développer.

Dans 40 p. 100 des prélèvements un Rotylenchus non décrit est associé à l'espèce précédente. Il se différencie aisément des autres Rotylenchus décrits par sa très courte taille, 0,47-0,62, malgré un stylet relativement long, 26-29,5 microns et des phasmides adanales. Moins abondant et de répartition plus restreinte que le Scutellonema, ce nématode peuł parfois dépasser le nombre de mille individus par $100 \mathrm{~g}$ de sol.

Il est aussi en plus grand nombre dans les échantillons de surface.

Une autre espèce nouvelle du même genre reste cantonnée dans un site de lande humide.

Enfin Helicotylenchus californicus Sher, 1966 a été identifié dans un prélèvement en lande humide. C'est la première espèce bisexuée du genre que l'on trouve en France.

L'autre groupe important, par le nombre et la dispersion, est constitué par sept Criconematidae représentés par : quatre Macroposthonia, un Hemicriconemoides, un Hemicycliophora et un Criconema.

Trois des quatre espèces de Macroposthonia sont communes en France : $M$. xenoplax (Raski, 1952) De Grisse et Loof, 1965, M. curvata (Raski, 1952) De Grisse ef Loof, 1965 ef M. rotundicauda (Loof, 1964) De Grisse et Loof, 1965 ; M. crenata (Loof, 1964) De Grisse et Loof, 1965, au contraire, n'y a jamais auparavant été signalé.

L'Hemicriconemoides n. sp. est voisin de H. cocophilus (Loos, 1949) Chitwood et Birchfield, 1957, dont il ne se distingue que par la forme de la tête. II n'est présent qu'en petit nombre dans les sites très secs et notamment dans les dunes. C'est la première fois que ce genre est trouvé en France. Toutefois, une espèce, $H$. pseudobrachyurum De Grisse, 1964, a été décrite en Belgique.

Criconema aquitanense Fies, 1966 n'a jamais jusqu'ici été trouvé ailleurs que dans les Landes, où se situe sa localité type. Il est répandu dans tous les paysages même dans les dunes, sauf cependant dans les zones les plus humides.

Hemicycliophora thienemanni (W. Schneider, 1925) Loos, 1948 est assez répandu en France. On ne le décèle ici que dans les sites très humides ef le plus souvent au-dessous de $20 \mathrm{~cm}$ de profondeur.

Parmi les Dorylaimida, les deux genres phytophages retrouvés sont Trichodorus ef Paratrichodorus.

Trois espèces ont été identifiées : T. similis Seinhorst, 1963, T. velatus, Hooper, 1972 et Paratrichodorus (Atlantadorus) pachydermus (Seinhorst, 1954) Siddiqi, 1974.

Par ailleurs une nouvelle espèce, appartenant au groupe Paratrichodorus, a été retrouvée dans des sites très éloignés géographiquement, mais toujours en faible nombre, ce qui tend à prouver que sa dissémination doit être plus étendue qu'elle ne paraît l'être à l'examen des premières analyses.

Aucun représentant des genres Xiphinema et Longidorus n'a jusqu'ici été décelé au cours de la prospection. 


\section{VI. - Etude de la répartition spatiale et des variations saisonnières des nématodes}

Avant de rechercher les concordances écologiques possibles abordées au chapitre suivant, nous avons essayé de faire la part des causes non liées au milieu qui pouvaient provoquer des modifications quantitatives ou qualitatives de la nématofaune ef de rechercher les horizons susceptibles de fournir le maximum d'espèces. Deux facteurs seulement ont pu être étudiés : les fluctuations saisonnières, la distribution verticale.

Enfin, nous avons voulu savoir si le dépérissement du pin maritime engendrait des modifications notables dans la nématofaune, afin d'isoler cet élément de variation des interprétations mésologiques. Nous n'évoquerons que très brièvement l'aspect du rôle de la nématofaune sur le développement du pin maritime. II fera l'objet d'une mise au point quand des éléments d'interprétation suffisants seront réunis.

\section{Fluctuations saisonnières}

Suivie pendant près de deux ans par des prélèvements trimestriels dans une des stations, l'étude des fluctuations de populations a don né lieu aux observations suivantes.

Parmi les Tylenchides :

Scutellonema n. sp. demeure en nombre élevé dans les couches supérieures - 5 à $20 \mathrm{~cm}$ - durant toute l'année et, bien qu'en moins grand nombre, il est également très constant entre 20 et $40 \mathrm{~cm}$.

II en est de même des Rotylenchus.

Les Criconematinae présentent un minimum d'avril à septembre et un maximum hivernal.

Les Mononchides sont plus fréquemment rencontrés en hiver et au printemps qu'en été et automne.

Ces données qui doivent être confirmées ultérieurement permettent le choix des espèces les plus stables tout au long de l'année, c'est-à-dire les moins influencées par les alternances climatiques. A cet égard Scutellonema n. sp. apparaît le nématode le plus intéressant.

\section{Distribution verticale}

Une analyse d'échantillons, prélevés dans chaque horizon en deux points voisins se différenciant principalement par le degré d'hygromorphie, révèle (tabl. 2) deux zones de concentration de la nématofaune : l'horizon $A 1$, humifère à forte densité racinaire, ef l'horizon $\mathrm{Bh}$, très noir, d'accumulation de la matière organique.

Cette concentration paraît assez indépendante de la profondeur puisque l'un des deux points de prélèvement présente un horizon intermédiaire $A 2$ lessivé très pauvre en nématodes.

On constate aussi que I'horizon A 1 renferme la quasi totalité des Scutellonema alors que c'est dans l'horizon Bh que l'on trouve la plus forte proportion des Criconematidae (Hemicycliophora et Macroposthonia). Il en est de même pour les Mononchides 
(prédateurs). Les saprophages se répartissent en proportions comparables dans les deux horizons précités.

Pour l'ensemble des autres sites échantillonnés à deux profondeurs différentes, généralement situés dans l'horizon A 1 , les prélèvements à $20-40 \mathrm{~cm}$ de profondeur se révèlent dans l'ensemble moins riches que ceux de surface $(-5 \mathrm{à} 20 \mathrm{~cm})$. Cette pauvreté relative se retrouve, à quelques exceptions près, dans tous les groupes trophiques et s'explique en partie par la grande richesse en matière organique des couches supérieures qui autorise un meilleur peuplement des espèces saprophages et bactériophages.

Action du dépérissement sur la nématofaune

Dans les zones dépérissantes, la nématofaune est moins riche ef les espèces phytophages moins nombreuses. Il est encore prématuré d'interpréter ce phénomène qui peut-être la cause ou la conséquence du mauvais développement du couvert végétal. Une série d'épreuves est destinée à préciser les rapports entre quelques-uns des principaux nématodes ef Pinus pinaster Sol.

\section{VII. - Concordances écologiques entre les «paysages landais » et la nématofaune}

Du point de vue mésologique, les six principaux types de « paysages » différenciés présentent de fortes analogies en raison précisément de l'unité de l'ensemble du territoire étudié. Toutefois, il est possible de discerner des particularités non négligeables au niveau de chacun des paysages (tabl. 1).

1) La lande humide est la plus riche en nombre et espèces de nématodes, ce qui tend à montrer que ce type de milieu est le plus proche du climax. L'abondance particulière de Scutellonema $n$. sp., endémique à la région landaise, renforce cette opinion.

2) La dune récente est la plus pauvre à cetégard et on y trouve la faune phytophage la plus réduite, ce qui s'explique peut-être par le petit nombre d'espèces végétales associées au pin qu'on y rencontre.

3) La lande sèche occupe une position intermédiaire. Elle est aussi plus riche que les zones semi-humides où les Tylenchides sont en très faible nombre. Ce fait est remarquable pour Scutellonema n. sp., mais n'a pu jusqu'à présent être expliqué.

Sur le plan de la signification écologique de la nématofaune, on relève un certain nombre de corrélations qui, dans cet exposé, a été limité aux groupes trophiques.

\section{Les saprophages-bactériophages}

Dans les sols squelettiques des dunes, où les teneurs en argile et en matière organique sont très faibles, on constate une abondance relative d'espèces de ce groupe.

En dehors des Plectus et des Panagrolaimus présents dans une forte proportion de prélèvements, les Wilsonema moins ubiquistes ne se retrouvent que dans les dunes. 


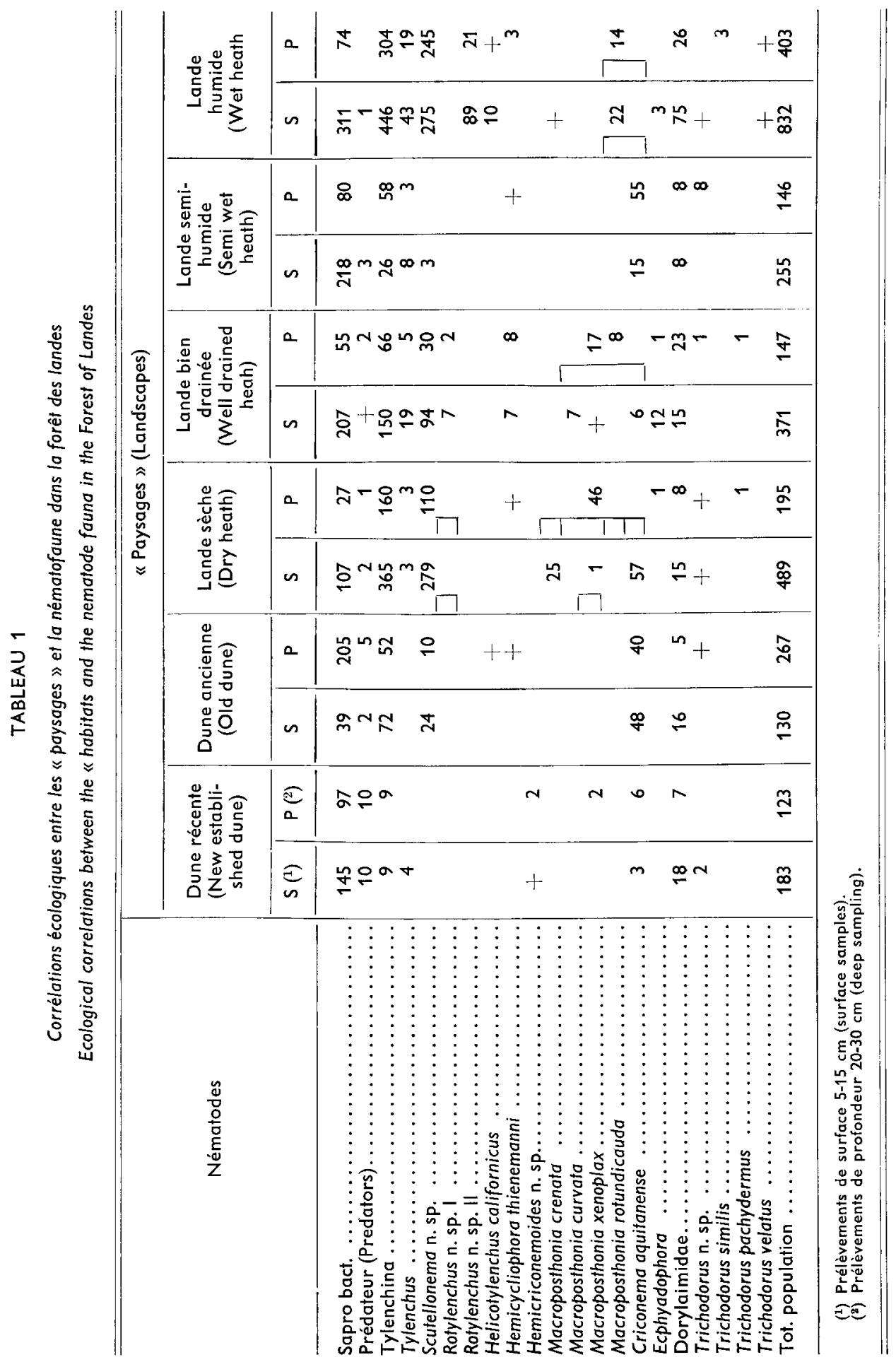


Si la localisation en surface est préférée dans les dunes récentes, c'est au contraire au-dessous de $20 \mathrm{~cm}$ que l'on retrouve le plus de bactériophages dans les formations dunaires plus anciennes, ceci est assez bien en rapport avec l'horizon d'accumulation de l'humus dont la profondeur est fonction de l'ancienneté de la colonisation du sol.

Dans les landes le nombre de bactériophages est toujours faible dans les sites les plus secs, leur nombre s'élève sensiblement quand l'humidité du sol augmente.

Dans tous les cas les horizons de surface sont plus riches que ceux situés audessous de $20 \mathrm{~cm}$. Aux Panagrolaimus et aux Plectus retrouvés à peu près partout se mèlent alors des Cephalobus et des Monhysteridae.

\section{Les prédateurs}

Le nombre de prédateurs est élevé dans les «dunes récentes », aussi bien en surface qu'en dessous de $20 \mathrm{~cm}$. Ce groupe perd de l'importance dans les « dunes anciennes ». Cette tendance s'accentue dans les «landes » au fur et à mesure que les paysages deviennent plus humides.

Presque uniquement composé de Mononchus dans les «dunes», ce groupe se diversifie dans les «landes » où aux Mononchus s'associent des Miconchus, des lotonchus et dans certains sites des Trypila, genre qui domine dans les endroits les plus humides.

Enfin en Gironde et dans deux sites de landes très excentrés, l'un en Vendée, l'autre en Dordogne, on a décelé en assez grand nombre Choanolaimus psamnophilus. Ce nématode prédateur a été décrit en 1880 par De Man d'après des exemplaires trouvés, probablement en Hollande, au pied de Gallium et de Sedum dans des sables dunaires littoraux ; il n'a été cité depuis qu'en 1960 par Chiłwood après examen de matériel récolté par Raski sur une plage de la côte océanique californienne.

Un des sites landais est situé à près de $100 \mathrm{~km}$ à l'intérieur des terres, ce qui remet en cause son caractère marin reconnu jusqu'ici.

\section{Les mycophages}

L’importance des mycophages est difficile à apprécier, car ce groupe est constitué de nombreuses espèces appartenant à des genres qui renferment aussi des nématodes phytophages : Tylenchus s. I., Nothotylenchidae, Aphelenchus, Aphelenchoides, etc. De plus, la destruction des champignons du sol peut avoir soit un rôle bénéfique en limitant le développement des cryptogames phytopathogènes, tel est, selon Riffle (1973), le cas de deux Aphelenchoides qui réduisent les dégâts d'Armillaires sur Pinus ponderosa Dougl. ; mais aussi des effets négatifs quand elle s'adresse aux mycorhizes associées aux conifères (Sutherland et Fortin, 1968).

\section{Les phytophages}

Les phytophages occupent un rôle primordial dans la nématofaune par le nombre et par la diversité des espèces. Ces deux derniers caractères croissent de façon accusée de la dune à la lande. Particulièrement rares el peu variés dans les dunes récentes où ils sont pratiquement inexistants, ils sont plus nombreux dans les dunes anciennes ef 
occupent une place de plus en plus importante lorsque l'humidité croît dans la lande. Or, ce dernier facteur constitue, rappelons-le, un élément déterminant dans la densité et le nombre des végétaux du sous-bois. II semble, de plus, qu'il y ait une relation plus étroite entre le couvert végétal ef la némałofaune phytophage, qu'entre cette dernière et le pin maritime présent dans tous les sites prospectés.

TABLEAU 2

Répartition selon les horizons pédologiques de la nématofaune de deux sites voisins en lande humide Distribution of the nematofauna according to the pedological horizon in two neighboring stations in wet lande

\begin{tabular}{|c|c|c|c|c|c|c|c|c|}
\hline \multirow{3}{*}{$\begin{array}{c}\text { Sites : } \\
\text { Horizons pédologiques } \\
\text { (Pedological horizons) }\end{array}$} & \multicolumn{4}{|c|}{ Site ! } & \multicolumn{4}{|c|}{ Site II } \\
\hline & \multirow{2}{*}{$\begin{array}{c}\text { humifère } \\
\text { A } 1\end{array}$} & \multirow{2}{*}{$\begin{array}{c}\text { lessivé } \\
\text { A } 2\end{array}$} & \multicolumn{2}{|c|}{ Accumulation } & \multirow{2}{*}{$\begin{array}{c}\text { humifère } \\
\text { A } 1\end{array}$} & \multicolumn{2}{|c|}{ Accumulation } & \multirow[b]{2}{*}{ Sable } \\
\hline & & & $\begin{array}{c}\text { M. } 0 . \\
\text { Bh }\end{array}$ & $\begin{array}{c}\mathrm{Fe} \\
\mathrm{BFe}\end{array}$ & & $\begin{array}{c}\text { M. o. } \\
\text { Bh }\end{array}$ & $\begin{array}{c}\mathrm{Fe} \\
\mathrm{BFe}\end{array}$ & \\
\hline $\begin{array}{l}\text { Profondeur en } \mathrm{cm} \text { (Depth in } \mathrm{cm} \text { ) } \\
\text { Population totale sur } 100 \mathrm{~g} \text { (Total } \\
\text { population for } 100 \mathrm{~g} \text { soil) } \\
\text { Saprophages }\end{array}$ & $\begin{array}{c}0-40 \\
\text { (a) } 251 \\
\text { (b) } 60,0 \\
85 \\
574\end{array}$ & $\begin{array}{l}40-52 \\
20 \\
4,8 \\
10 \\
6,8\end{array}$ & $\begin{array}{l}52-62 \\
135 \\
32,3 \\
45 \\
304\end{array}$ & $\begin{array}{l}60-110 \\
12 \\
2,9 \\
8 \\
54\end{array}$ & $\begin{array}{l}0-30 \\
75 \\
33,5 \\
28 \\
45\end{array}$ & $\begin{array}{l}30-40 \\
131 \\
58,5 \\
28 \\
45\end{array}$ & $\begin{array}{c}40-75 \\
18 \\
8,0 \\
6 \\
0\end{array}$ & $\begin{array}{l}75 \\
+ \\
+\end{array}$ \\
\hline Mononchides & - & - & $\begin{array}{l}10 \\
71,4\end{array}$ & $\begin{array}{c}4 \\
28,6\end{array}$ & - & $\begin{array}{c}6 \\
100,0\end{array}$ & - & - \\
\hline Tylenchida & $\begin{array}{c}159 \\
63,9\end{array}$ & $\begin{array}{l}10 \\
4,0\end{array}$ & $\begin{array}{l}80 \\
32,1\end{array}$ & $\overline{19,5}$ & $\begin{array}{c}25 \\
71,1\end{array}$ & $\begin{array}{l}91 \\
71,1\end{array}$ & $\begin{array}{c}12 \\
9,4\end{array}$ & + \\
\hline Tylenchus spp. & - & - & $\begin{array}{c}25 \\
100,0\end{array}$ & - & - & $\begin{array}{c}6 \\
100,0\end{array}$ & - & - \\
\hline Scutellonema n. sp. & $\begin{array}{l}135 \\
100,0\end{array}$ & + & $\begin{array}{l}+ \\
+\end{array}$ & - & - & - & - & - \\
\hline Hemicycliophora thienemanni & $\begin{array}{l}24 \\
27,0\end{array}$ & $\begin{array}{l}10 \\
11,2\end{array}$ & $\begin{array}{l}55 \\
61,8\end{array}$ & - & $\begin{array}{l}25 \\
25,3\end{array}$ & 62 & $\begin{array}{c}12 \\
12,1\end{array}$ & - \\
\hline Macroposinonia xenoplax & & 一 & - & - & - & $\begin{array}{c}23 \\
100,0\end{array}$ & - & - \\
\hline Dorylaimida divers & $\begin{array}{l}+ \\
+\end{array}$ & $\begin{array}{l}+ \\
+\end{array}$ & $\begin{array}{l}+ \\
+\end{array}$ & - & $\begin{array}{l}19 \\
76,0\end{array}$ & $\begin{array}{c}6 \\
24,0\end{array}$ & - & - \\
\hline Trichodorus spp. & - & - & - & - & $\begin{array}{c}3 \\
100,0\end{array}$ & - & - & - \\
\hline $\begin{array}{l}\text { Espèces dominantes du sous-bois } \\
\text { (Main species of the underwood) }\end{array}$ & \multicolumn{4}{|c|}{$\begin{array}{l}\text { Pteridium aquilinum } \\
\text { Molinia coerulea } \\
\text { Ulex nanus } \\
\text { Erica scoparia }\end{array}$} & \multicolumn{4}{|c|}{$\begin{array}{l}\text { Molinia coerculea } \\
\text { Erica tetragona } \\
\text { Potentilla sp. } \\
\text { Calluna vulgaris }\end{array}$} \\
\hline $\begin{array}{l}\text { Hydromorphie } \\
\text { (Hydromorphy) }\end{array}$ & \multicolumn{4}{|c|}{$\begin{array}{l}\text { Modérée } \\
\text { (Moderate) }\end{array}$} & \multicolumn{4}{|c|}{$\begin{array}{l}\text { Importante } \\
\text { (Heavy) }\end{array}$} \\
\hline
\end{tabular}

(a) 1 re ligne : nombre d'individus pour $100 \mathrm{~g}$ de sol.

(b) 20 ligne : p. 100 par rapport à l'ensemble des autres horizons de chaque profil.

(a) Number of nematodes $/ 100 \mathrm{~g}$ soil.

(b) P. 100 of all four horizons of each pedological profil.

Les nématodes phytophages appartiennent à deux ordres : les Tylenchida ef les Dorylaimida. Le premier renferme l'espèce la plus répandue.

- Scutellonema n. sp., qui occupe tous les sites des plus humides aux plus secs à l'exception de la dune récente. Ce nématode ne paraît pas se multiplier sur pin, mais 


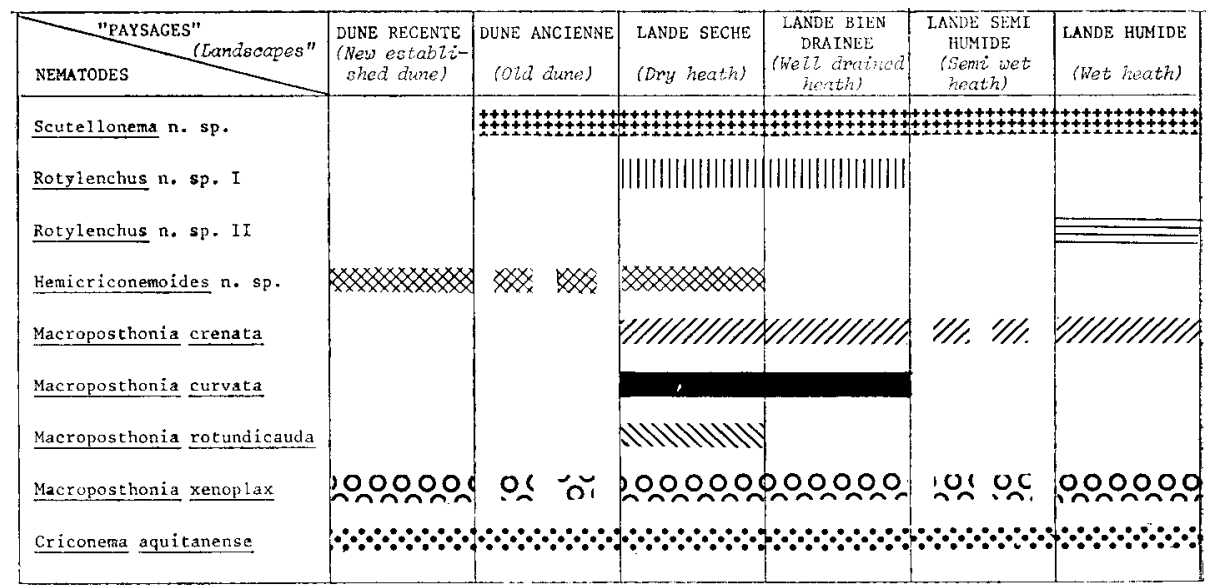

FIG. 2. - Corrélations écologiques entre les « paysages 》 ef quelques espèces de nématodes phytophages dans la forêt des Landes.

(Ecological correlaftions between "landscapes " and some species of phytophagous nemaiodes in Landes forest).

plutôt sur un ou plusieurs des végétaux du sous-bois. Cette opinion est confortée par le fait que les tentatives pour le multiplier sur Pinus pinaster en pot ont jusqu'ici échoué : de plus la destruction du sous-bois, recommandée dans les peuplements de rapport, entraîne sa diminution.

- L'autre espèce, la plus disséminée comme la plus abondante après la précédente, est Criconema aquitanense, également originaire de la région landaise. On la retrouve, bien qu'en très faible nombre, dans les dunes récentes, mais elle est absente des sites plus humides. Elle manifeste un préférendum pour les sols bien aérés. Ce caractère est encore plus marqué pour Hemicriconemoides n. sp., qui ne se retrouve que dans les dunes récentes en landes sèches.

Les quatre Macroposthonia identifiés coexistent dans la lande sèche ; mais si $M$. rotundicauda n'a pu être décelé ailleurs, $M$. curvata se retrouve dans la lande bien drainée, $M$. crenata dans les zones les plus humides et $M$. xenoplax a été isolé aussi bien dans les dunes récentes que dans la lande humide.

\section{VIII. - Conclusion}

Les premiers résultats de l'inventaire nématofaunistique qui fait l'objet de cette étude illustrent :

L'unité synécologique du massif landais par la présence d'espèces identiques dans la plupart des sites échantillonnés.

L'originalité particulière de cette région marquée par la découverte de plusieurs espèces nouvelles et de deux genres inconnus à ce jour dans le reste de la France.

La faible influence du pin maritime sur la nématofaune phytophage, ce qui confère au sous-bois un rôle déterminant dans l'alimentation ef la multiplication des espèces 
de ce groupe. Cette dernière observation met bien en évidence le caractère endémique du couvert végétal par rapport au Pinus pinaster introduit.

On observe dans l'ensemble des résultats obtenus une certaine corrélation entre le développement du pin et le nombre de nématodes phytophages, mais les rapports liant les nématodes et le couvert végétal ne pourront être expliqués qu'après une série d'épreuves en cours.

L'influence déterminante du degré d'évolution pédologique des sols ef de leur hydromorphie sur la structure des populations se dégage d'ores et déjà des premiers éléments de cette étude. La prise en considération d'autres éléments des écosystèmes éfudiés : édaphiques et phytosociologiques, est projetée grâce à la participation des spécialistes du groupe de travail «Equilibre biologique et Productivité en forêf landaise $)$.

Enfin une carłographie nématologique du massif landais est à l'étude, elle doił être à la base d'un essai de concordances avec certaines données écologiques.

Ces travaux devraient permettre de mieux saisir le déterminisme de la répartition spatiale de ce groupe zoologique, ainsi que les exigences de chacune des espèces concernées.

Les nématodes représentant, comme l'ont rappelé Ferris, et al. (1972), les métazoaires les plus nombreux des habitats telluriques naturels, il importe d'en approfondir l'étude pour préciser leur rôle au sein des bioceonoses.

Nous sommes persuadés qu'une meilleure connaissance des exigences trophiques et écologiques des représentants de ce groupe ferait de l'analyse nématologique un moyen très performant de diagnostic écologique des milieux telluriques naturels.

Reçu pour publication en avril 1979.

\section{Summary}

Ecological significance of nematofauna of the «Landes » forest in south west of France

A nematological survey of the forest clad of the "Landes » shows an exceptional originality and a relative homogeneity of this site.

The original feature is the almost exclusive presence of some genera of Hoplolaiminae and Criconematinae. Four species of the Tylenchida are new; one Scutellonema genus which is first reported in Europe and one species of each genus Rolylenchus, Helicotylenchus, Hemicriconemoides, Criconema aquitanensis is already described. There is a new Trichodorus too.

Homogeneify expresses the large widespreading of some species found in the most of the samples, this cannot be explained by the action of men.

These two characteristics result from the pedological constitution and from phytosociological and climatic features of the area : this area orginated from sand hills is constituted by a no calcareous sandy horizon of variable thickness and granulometry. The whole forest is submitted to the typical atlantic climate and is grown since the beginning of this century, only with Pinus pinaster associated with a variable plant clad on a surface of one million of hectares.

In this large area edaphic and agropedologic characteristics look to have a more important influence than climatic and phytosociologic ones on the nematofauna, many of its species being able to survive on other crops when introduced in this region. 


\section{Resumen}

Significacion ecologica de la nematofauna del pino maritimo en el bosque landes en el sud-oeste de Francia

Una prospeccion nematologica en el macizo landes, revela una exceptional originalidad y una cierta homogeneidad, de este lugar.

La originalidad se traduce en particular, por la existencia casi exclusiva de algunos generos de Hoplolaiminae y de Criconematidae y por la presencia de 5 nuevas especies de tylenchides : Scutellonema, este genero es senalado por primera ver en Europa, Rofylenchus, Helicotylenchus, Hemicriconemoides, Criconema aquitanensi y de una especie de Trichodorus.

La homogeneidad, explica la gran extension de algunas especies encontradas en la mayor parte de las tomas, que no puede justificarse por la accion del hombre.

Estas dos caracteristicas resultan de la consfitución pedologica y de los factores, climatico y fitosociologico de esta region.

Del punto de vista pedológico, es una zona de dunas, constituida por un horizonte arenose no calcareo de espesor y granulometria variable. El clima aflantico influye todo el macizo landes, y el pino maritimo Pinus maritima asociado a una cubierta vegetal variable, ocupa casi complemente 1 million de hectareas desde cumienzos de siglo.

En esta vasta zona, los caracteres edaficos y agropedologicos del lugar, parecen tener una influencia más marcada que los otros dos factores sobre la naturaleza y reparticion de la nematofauna.

\section{Références bibliographiques}

BAUJARD P., 1975. Essai d'interprétation de la répartition des groupes trophiques de la nématofaune dans un secteur du massif landais. Mémoire, thèse, 130 p. (non publié).

DEMOUNEM R., 1968. Ecophysiologie végétale. Influence du type de sol forestier sur la croissance des espèces arbustives du sous-bois des Landes de Gascogne. C. r. Acod. Sci., 1501-1504.

FIESM., 1966. Criconema aquitanense n. sp. Nematologica 14,47-54.

GUINAUDEAU J., 1973. La forêt landaise. Doc. I.N.R.A., Ronéo, 45 p.

RIFFLE J.W., 1973. Effect of two mycophagous nematodes on Armillaria mellea root rot of Pinus ponderosa seedlings. Plant Dis. Reptr., 57, 355-357.

SUTHERLAND J. R. ef FORTIN J. A., 1968. Effect of the nematode Aphelenchus avenae on some ectotrophic mycorrhizal fungi and on a red pine micorrhizal relationships. Phytopathology, $\mathbf{5 8}$ 519-523.

FERRIS V. R., FELDMESSER J., HANSEN E., LEVINE N. ef TRIANTAMHYLLOU A. C., 1972. Features and news. The importance of discoveries in nematology to human welfare. Bio. Science, 22, 237-239. 